\title{
Robot Integrated User Interface for Physical Interaction with the DLR MIRO in Versatile Medical Procedures
}

\author{
C. Schlenk, T. Bahls, S. Tarassenko, J. Klodmann, M. Bihler, T. Wuesthoff \\ Institute of Robotics and Mechatronics, German Aerospace Center (DLR), 82234 Wessling, Germany \\ E-mail: christopher.schlenk@dlr.de
}

To enhance the capability of the DLR MIRO for physical human robot interaction (pHRI) six buttons were integrated as additional input interface along the robot structure. A ring of eight RGB-LEDs at the instrument interface informs the user as additional output interface about the robot's state. The mechatronic design, which is transferable to other robots, adapts to the existing communication infrastructure of the robot and therefore offers real-time capability. Besides the interaction with the robot itself it also allows the control of 3rd party devices connected to its communication network. Both interfaces can be flexibly programmed e.g. in $\mathrm{C}++$ or Simulink.

Keywords: physical human robot interaction; lightweight robot; hands-on robotics; DLR MIRO; real-time capable buttons; RGBLED.

\section{Introduction}

In the last two decades, commercial medical robots have become established tools in various medical fields like cancer therapy (e.g. CyberKnife [Accuray Inc., Sunnyvale, CA, USA]) [1], minimally invasive surgery (e.g. da Vinci [ntuitive Surgical Inc., Sunnyvale, CA, USA])[1], orthopedics (ROBODOC, [THINK Surgical Inc., Fremont, CA, USA]) [1], spine surgery (Renaissance and since 2016 Mazor X, [Mazor Robotics Ltd., Caesarea, Israel])[1, 2] and neurosurgery (NeuroMate, [Renishaw plc., New Mills, UK]) [2].

The CyberKnife system for cancer therapy exploits the robot's ability for performing autonomously precise, reproducible movements to radiate tumor tissue according to a previously developed treatment plan.

In minimally invasive robotic surgery (MIRS), the robotic system reduces the complexity of the surgical procedures for the surgeon while preserving their advantages for the patient. This application utilizes the robot's ability to control additional degrees of freedom inside the patient and restore the surgeon's hand-eye coordination.

In orthopedics, $R O B O D O C$ makes use of the accuracy and stiffness of the robotic arm to autonomously mill cavities in the patient's bone.

The Renaissance robot for spine surgery orients the manually moved surgical tool towards the target area defined in the preoperative planning. The guide rail, along which the robot arm moves, is screwed directly to the patient's spine. This prevents any relative movement between the robot and the patient [1].

The NeuroMate robot pursues a similar approach in the field of neurosurgery. The patient position with regard to the robot is registered either by connecting the robot to the stereotactic frame at the patient's skull or by registering both patient and robot using an external tracking device. Subsequently the robot arm can ensure the tool orientation towards the desired target area. The tool itself e.g. an electrode or a biopsy needle — is manually inserted by the surgeon.

All the above mentioned named systems are during the surgical intervention either teleoperated (e.g. da Vinci) or perform their tasks autonomously according to a preoperative plan. Each of these commercially available robots is specialized for particular medical scenarios. In combination with the high system prices (according to [1] a da Vinci Si cost more than $\$ 1.5 \mathrm{M}$ in 2014 ), this hinders the use of such systems outside of large hospitals. Additionally most commercially available systems are rather bulky (an exception is the patient-mounted Renaissance system). Their high weight - e.g. $180 \mathrm{~kg}$ for the NeuroMate [3] and $821 \mathrm{~kg}$ for the patient cart of the da Vinci Xi[4] - makes the movement of the robotic system within the OR laborious. Systems like $d a$ Vinci also consume much space around the OR table [1], which may impede the work of the medical staff at the OR table.

A promising, evolving alternative are versatile robotic systems, which can perform a broad range of medical tasks. These systems may enable also small hospitals with com- 
paratively low patient numbers to benefit from the new technology and thereby contribute to the democratization of robotic surgery. Compact lightweight robots like the DLR MIRO (see Fig. 1) [5, 6] or the KUKA iiwa [KUKA AG, Augsburg, Germany] are an appropriate base for such versatile robotic systems: Firstly, they allow due to their integrated torque sensors a safe interaction with humans (cp. [7]). This is crucial in the medical field, where the patient and often also the medical staff are located within the workspace of the moving robot. Secondly, they provide besides autonomous operation and teleoperation also the possibility for physical human robot interaction (pHRI). In this control mode (in [6] named hands-on control), the human user interacts physically with the structure of the robot arm to move it in a desired way. This control mode may be beneficial in various scenarios:

- It simplifies and accelerates the setup of complex robotic systems, e.g. in MIRS. Since the positioning of the robot arm can be realized by direct motion of the robot, it is both intuitive and easily parallelizable.

- For autonomous and teleoperation systems it enlarges the possibilities of the operating room staff at the OR table to interact with the robot. Since the control of the robot is not exclusively bound to the surgeon console any more, routine procedures like an instrument change in MIRS or a nullspace motion of the robotic arm to improve patient access for the staff can be performed autonomously by the OR staff. This disburdens the surgeon in routine procedures and allows him to focus on his task.

- In various medical applications using a single robot arm, e.g. robotic waterjet surgery [8], vessel detection using doppler ultrasonography [9], placement of pedicle screws [10] and biopsy needles [11] or robotic neuroendoscopy [12], pHRI might even make an additional input device obsolete. This would not only simplify the system setup but also improve the usability, since the user would not have to interact with both the robot arm itself and an input device.

From the technical viewpoint all the above described scenarios require a smooth switching of the robotic control mode (e.g. from pHRI for setting up the MIRS system to teleoperation during the surgical intervention) on demand. The most straightforward way for switching control modes are direct user commands, which the robotic system recognizes as events. During pHRI the user is already in physical contact with the robot. Thus the integration of manually operated buttons in the robot arm offers a cost-efficient, space-saving, ergonomic and reliable possibility for receiving these user commands. The buttons must enable the user to navigate through a predefined workflow (by choosing and confirming workflow steps) and also to trigger particular events like e.g. an instrument change. To report the consequences of the user actions, additionally an appropriate output interface is required. This output interface should also be integrated in the robot to minimize the size of the robotic setup.

Several commercially available robotic systems contain user interfaces consisting of buttons as input and LED rings as output interface or allow pHRI.

The two-armed robotic worker Baxter [Rethink Robotics, Boston, MA, USA] possesses an input interface consisting of a knob and two buttons at each arm and displays its system state via an LCD and a LED ring at its head [13]. Its successor, the robot arm Sawyer, also includes an input interface with a knob and five buttons and the same output interfaces [14].

The KUKA iiwa, which is used both in industry and in many research applications, can be equipped with a button and a LED ring at the tool interface. $K U K A$ demonstrated in a video [15], how this interface can be used for teaching the trajectory in a pick-and-place task.

The RIO system [Stryker Inc., Kalamazoo, MI, USA] for hip and knee surgery provides an intraoperative handson control of the the robotic arm by the surgeon. Based on patient CT data and preoperative planning, the robotic arm limits the movements, which the surgeon performs with the attached milling tool, to the predefined workspace using virtual fixtures [1]. However, the robot arm itself includes no user interfaces. Instead the system is controlled from a separate control cart.

The Mazor $X$ system for spine surgery, which supports the surgeon by orienting the drill guidance according to the preoperative plan, displays its status using an LED ring at the tip of the robot arm but has no input interface integrated in the arm. The robotic arm is controlled via a touchscreen on its cart [16].

The daVinci Xi system for MIRS offers at each of its patient cart arms buttons for gravity compensated motion and motion around the trocar point. Additionally each arm includes four LEDs to indicate its status [4].

For a researcher, however, who necessitates extensive reconfiguration possibilities to implement new applications, these commercially available solutions have some drawbacks:

- Limited flexibility: Reprogramming is limited or not possible. This obstructs the adaptation of the robotic system to new applications.

- Unknown signal round trip time: High signal roundtrip times may cause problems when e.g. the control mode of a robotic arm shall be switched.

- Do not allow control of 3rd party devices connected to the robotic system. "To ensure interoperability of systems", however, is according to [1] currently a central challenge in medical robotics.

In medical robotics research, several publications have suggested a synergistic surgeon-robot cooperation, connecting the strengths of humans (information management, decision-making, legal responsibility etc.) and robots (accuracy, strength, reproducibility etc.) $[11,12,17]$. In [12], for example a shared control approach for the insertion of a neuroendoscope is described, in which an $K U K A L W R$ 
supports the surgeon in the insertion procedure by providing virtual walls. For the further development of such approaches the existence of adequate workflows and interaction devices is crucial. However, while the integration of LEDs for displaying the system state or even guiding the user (as described for a non-robotic system in [18]) is common, most medical robotics research systems still use separate input devices like foot pedals, tablets or remote control consoles (see for example [12]) for controlling the robot.

Therefore in this work a new user interface for pHRI in medical robotics research is presented, which overcomes the above-named drawbacks of the commercially available solutions and is compact and light enough to be integrated in a state of the art lightweight robot.

In the following we present the integration of this new user interface into the robotic research platform $D L R$ $M I R O$. It consists of six programmable, real-time capable buttons along the robotic structure as additional input and a ring of RGB-LEDs at the instrument interface as additional output interface. To illustrate its practical applicability we implemented workflows for a single-arm application (wound debridement using a waterjet) and a multiarm application (setup of a MIRS system). The usability of our approach was evaluated by a user test of the MIRS setup procedure. The following considerations may also be adapted and transferred to other medical robot research systems like the KUKA LWR or the daVinci Research Kit.

\section{Materials and Methods}
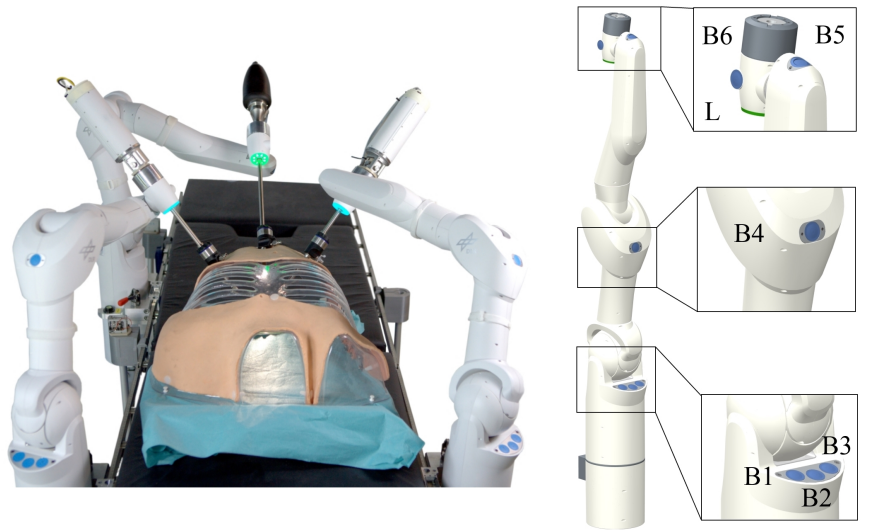

Fig. 1. Left: The DLR MiroSurge demonstration setup for MIRS: The $M I R O$ in the middle carries a stereo endoscope, the other two MIROs carry the robotic DLR MICA instruments (see [6]). The MICA instrument is an actuated, lightweight instrument for MIRS with two wrist degrees of freedom (DOF) and one end-effector DOF [6]. Right: Rendering of the MIRO with the buttons (B1-B6) and RGB-LED ring L.

The user interface consisting of six programmable, real-time (RT) capable buttons along the robotic structure and a ring of eight RGB-LEDs at the instrument interface was subsequently integrated in the MIRO robot arm (see Fig. 1).

With its low weight and integrated torque sensors this robot meets all the requirements for safe pHRI. The versatile robotic arm can be adapted to various applications by changing the attached specialized tools and modifying its high-level control software [compare 6, 8, 9, 10]. For investigating the potential of the new user interface, we chose the applications MIRS [6] and wound debridement using a waterjet [8], since they exemplify the wide range of applications the MIRO can be used for: A complex telemanipulation setup with three robot arms and a surgeon console vs. a semi-autonomous single-arm setup.

\subsection{Input interface: robot integrated buttons}

To simplify the handling of the MIRO in pHRI mode, the buttons were distributed along the robotic arm in such a way, that grasp areas for the user were defined. Based on a workshop with medical staff (five surgeons, one scrub nurse) and tests with DLR staff, the number and spatial arrangement of the buttons shown in Fig. 1 has been chosen as adequate for pHRI with the MIRO robot. The buttons B1, B2 and B3 form a menu panel to navigate through the workflow, if e.g. the functions backwards, confirm and forwards are assigned to them. With the buttons B4 and B5 the robot can be switched to particular control modes like gravity compensated freehand motion, impedance control parametrized w.r.t. application relevant constraints (e.g. trocar points in MIRS) or nullspace motion. Button B6 unlocks the robot's instrument interface.
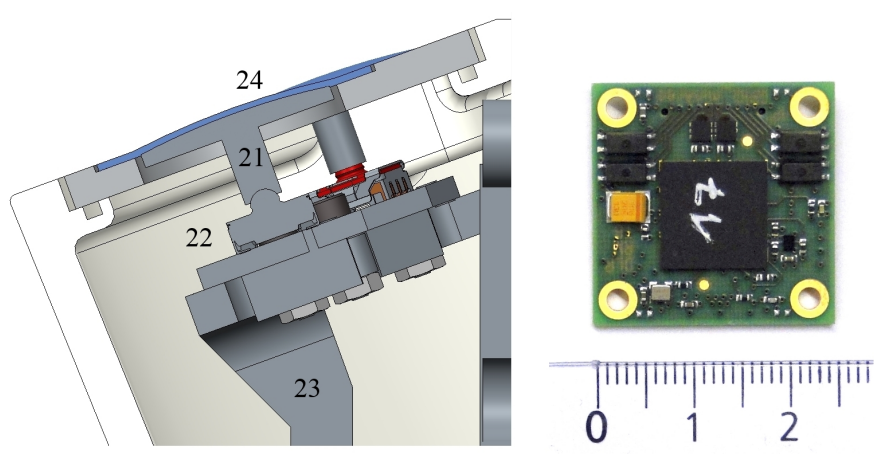

Fig. 2. Left: Mechanical structure of B2: The tappet (21) transmits the finger pressure to the switch (22), which is connected to the robot's mechanical structure by a RP part (23). A flexible membrane (24) covers the casing opening. Right: GPIO board with centimeter-scale.

The buttons are read in by a general purpose input output board (GPIO board). This $24 \mathrm{~mm}$ x $24 \mathrm{~mm}$ large board (see Fig. 2) provides nine single ended IOs, 
two galvanically insolated IOs and two power switches. Its FPGA technology enables data-reprocessing of several inputs/outputs in real-time parallel to the communication process and offers full flexibility regarding the communication protocol which forms the connection to the communication infrastructure of the robot arm. The RT capability of the GPIO board allows the direct allocation of the event pressing/releasing the button to the temporal representation of the robot. In the MIRO the integration into the real-time capable SpaceWire communication of the robot is achieved via a communication bridge to a proprietary protocol transmitted via three LVDS (Low Voltage Differential Signaling) lines. Alternatively any other communication protocol transmittable via three LVDS (e.g. BISS or 3 -wire SPI) can be used. In this case the FPGA firmware, which is currently created using generic VHDL, must be adapted accordingly.

The button design (see Fig. 2) provides distinct tactile feedback to the user (which is important for preclinical studies, in which the robot is draped), ensures mechanical robustness (as all sensitive components are integrated into the casings) and simplifies the (dis)assembly of the casings (as no disconnection of the cables is necessary to disassemble the casings).

Since the casings of the MIRO are no integral part of the robot structure, rapid prototyping $(\mathrm{RP})$ parts attach switch, tappet and GPIO board to the mechanical structure. The openings for the tappets in the casings are covered by a flexible membrane, which prevents the intrusion of dust or fluids and thereby allows the disinfection of the robot (when sterility is required, however, the robot must be wrapped in sterile drape).

As displayed in Fig. 3, the GPIO board samples the electrical signal of one or more (in the present installation up to three) buttons and feeds it into the RT communication of the robot. The control software of the robotic system considers the digital button signals as additional sensor inputs from the robot arm. The used communication protocols are shown in Fig. 5. More detailed information about the communication architecture of the MIRO can be found in $[19,20]$, since the hardware communication architectures of the DLR Hand Arm System and the MIRO follow the same principles. Thanks to the modular software architecture just minor software changes are required to integrate the support of the buttons. Only the software interface for the high-level control of the MIRO, the MIRO Hardware Abstraction Layer (HAL) [5], must be slightly modified: It is extended by a button interface via an additional Inter Process Communication Channel (IPCC). This channel is one-directional since data is only transmitted from the buttons to the robotic control system. To access the data from this $I P C C$, the software modules providing user interaction with buttons can use automatically generated functional stubs, e.g. a Matlab Simulink library [The MathWorks Inc., Natick, MA, USA] or a pre-compiled $\mathrm{C}++$ library.

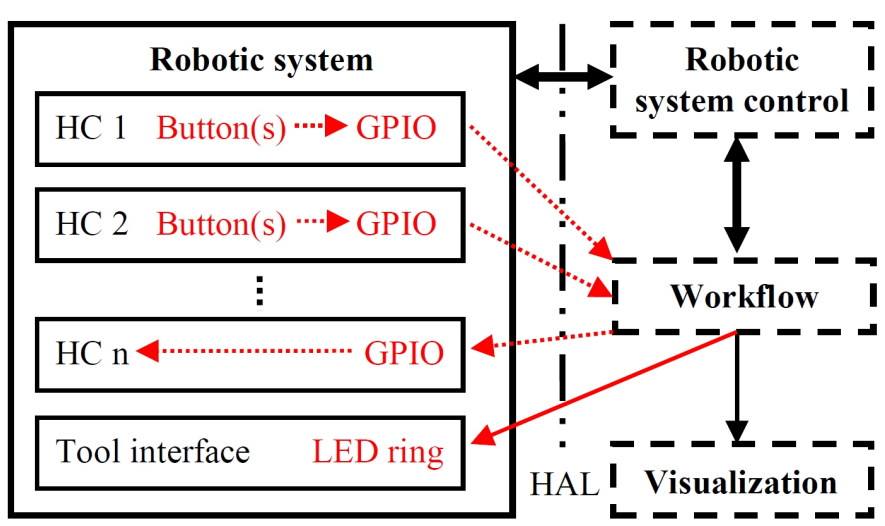

Fig. 3. The button(s) and the GPIO board together form an input interface, which can be attached to any hardware component $\mathrm{HC}$ i of the robotic system, e.g. the MIRO. It enables the user to navigate through the workflow without an additional input device, e.g. tablet computer or SpaceMouse [3DConnexion, Munich, Germany]. Depending on the workflow step the robotic system control and the system visualization in the virtual reality (VR) can be adapted. On the other hand, the GPIO board alone can serve as bridge between the software implemented workflow and a 3rd party hardware component $\mathrm{HC}$. The LED ring at the tool interface forms an additional output interface, which is directly integrated in the robotic system.

\subsection{Output interface: integrated ring of RGB-LEDs}

To inform the user without any external device about the system state of the robotic arm, a board with eight RGBLEDs APA102C [ShijiLighting, Shenzen, China] equally distributed around the tool shaft axis has been integrated at the instrument interface of the robot (see Fig. 4).
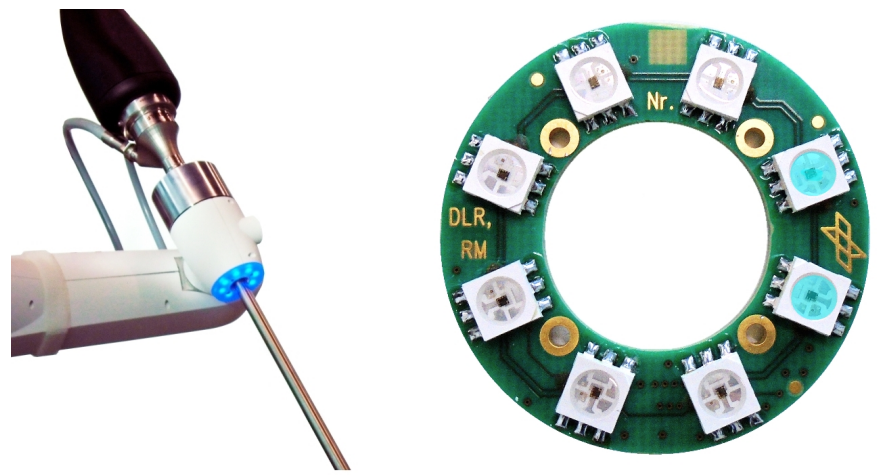

Fig. 4. Left: Detail view of the LED ring at the instrument interface of the MIRO. Right: Board with the eight RGB-LEDs APA102C. To guide the user in pHRI mode e.g. to the right, only the two right LEDs of the ring would be activated. 
The integrated controller enables the addressing of all eight LEDs via a generic 2-wire SPI interface, which is connected to the FPGA on the MIRO's digital electronics (DE) via two LVDS lines followed by a level switch from differential to single ended. A communication bridge in the FPGA's firmware integrates the SPI interface into the MIRO's SpaceWire communication (compare Fig. 5). Since the control of the LED ring is not critical with regard to the safety of the robotic system, a RequestResponse protocol [20] is used for its SpaceWire communication channel. Thus the LEDs are not reset automatically synchronous to the $3 \mathrm{kHz}$ clock domain but expect an appropriate command from the host.

The communication between the LED ring and the robotic system control uses - like the communication between buttons and the robotic system control - an additional $I P C C$ in the $H A L$. The only difference is the direction of the one-directional communication channel, since the data is sent from the robotic system control to the LED ring. That data is created using automatically generated functional stubs as described in subsection 2.1.

Since the LEDs can be adressed independently and with high frequency, different coding strategies can be implemented:

- Static coding: All LEDs have the same color and brightness to display e.g. the system state of the robot (an exemplary color scheme for visualizing the system state of the MIRO is displayed in Table 1). Typically this parameters remain unchanged for some time.

- Dynamic coding: The color and brightness of each LED is different and changes permanently depending on the user's actions. This coding allows e.g. to guide the system user in pHRI mode to a predefined position.

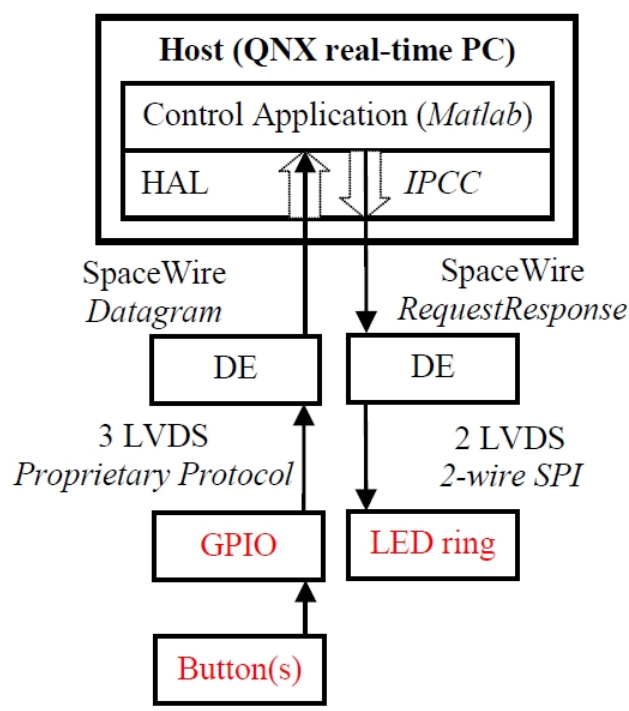

Fig. 5. Schematic representation of the communication channels for the buttons and the LED ring (compare [19, 20]). The GPIO board translates the electrical signal of the button(s) into a proprietary protocol and sends it via three LVDS lines to a digital electronics board (DE) of the MIRO. From there the signal is transmitted to the host via the SpaceWire communication of the robot arm using the Datagram protocol [20]. The host is a QNX real-time PC which runs the control application - for the MIRO a compiled Matlab model - and the $H A L$ with the additional $I P C C$ s for the button and LED ring signals (dotted arrows). To activate the LED ring, the host sends a signal through the SpaceWire communication of the robot arm using a RequestResponse protocol [20]. In the DE connected to the LED ring the signal is translated into $2-$ wire SPI. Then it is sent via two LVDS lines to the LED ring.

\subsection{Usability test: MiroSurge setup}

To verify the practical use of the new interface, we performed a usability test with 12 test persons ( 7 male and 5 female, age $30.8 \pm 9.0$ years). None of them had previous experience in using the robot integrated interface of the MIRO. Thus they received at first a general introduction to the MiroSurge system and an explanation of the button functions, which lasted about 10 minutes. Subsequently the test persons had about three minutes to familiarize themselves with the MIRO and the integrated interface by switching the control modes and moving the robot arm under supervision. After answering remaining open questions, the actual user test began: Each test person set up the threearm research platform MiroSurge displayed in Fig. 1 once with and once without using the robot integrated buttons. The respective setup time was measured and the subjective user experience for both scenarios was evaluated with the system usability scale [21]. To compensate for learning effects, six test persons started with the setup procedure using the robot integrated buttons, the other six with the setup procedure not using them. The statistical significance of the test results was checked by a two-sided paired t-test.

\section{Results}

In this section the claimed technical properties are evaluated and the implemented workflows for waterjet wound debridement and the setup of the MIRS research platform MiroSurge are described. Subsequently the results of the usability test are presented.

\subsection{Evaluation of technical properties}

In this subsection the RT capability, the control of 3rd party devices and the function of the LED ring are evaluated.

\subsubsection{RT-capability}

The RT capability of the GPIO board and its integration in the robot communication allows time-critical applications, 
e.g. control mode switching, where any uncontrolled motion of the robot must be prevented (e.g. when switching from pHRI to autonomous mode), or sampling surfaces to build a 3D model, where button toggling and joint positions of the MIRO must be registered synchronously w.r.t. time. The debouncing circuit for the buttons integrated on the GPIO board has a debouncing time of $1000 \mu \mathrm{s}$. After this time the button signal is recognized by the FPGA on the GPIO board and transmitted to a digital electronics board of the MIRO. Since the buttons can trigger safetycritical events, e.g. the change of the control mode of the robot, their state is provided cyclically via a hardware triggered Datagram communication synchronous to the $3 \mathrm{kHz}$ clock domain [20]. Therefore the maximum roundtrip time for the path digital electronics (DE), $H A L$, control application (compiled Matlab model on QNX real-time $\mathrm{PC}$ ), $H A L$, DE (see Fig. 5) is $333 \mu \mathrm{s}$. The real signal latencies in the MIRO are typically between $100 \mu$ s and $175 \mu$ s (compare $[5$, Figure 8]). This leads to a maximum button signal latency of $1666 \mu$ s for pressing and releasing the button (one communication cycle of $333 \mu$ s might get lost if the output register on the DE is just set while the register's value is interrogated). An asynchronous communication between GPIO board and host could further reduce the signal latency but is more critical with regard to safety. While in the cyclic communication the button state in the output of the GPIO board is reset in every cycle and thus an error is corrected after maximally $333 \mu \mathrm{s}$, an error in an asynchronous communication signal can only be recognized when a new signal is triggered.

\subsubsection{Control of 3rd party devices}

In addition to the robot, the buttons can also control 3rd party devices connected to the same communication network. In this case the GPIO board can be used to connect the 3rd party device ( $\mathrm{HC} \mathrm{n}$ in Fig. 3) with the communication network of the robotic system.

One example for this application of the buttons is the control of the commercially available waterjet and suction pump ERBEJET 2 ( [Erbe Elektromedizin GmbH, Tuebingen, Germany] via the GPIO board and the robot buttons. Before the implementation of the workflow for waterjet wound debridement described in subsection 3.2, the replacement of the normally used foot pedals by the GPIO board and Matlab Simulink was successfully tested. In the final workflow, the pump for the waterjet and the suction can be controlled directly by the robot integrated buttons.

\subsubsection{Function LED ring}

The LED ring was programmed to display the robot status via the same predefined color scheme, that is implemented in the virtual reality (VR) model of the MiroSurge system (compare Fig. 7):
Table 1. Color scheme for visualizing the system state of the MIRO at the LED ring and in the VR model.

\begin{tabular}{lc}
\hline System state & Assigned color \\
\hline Ready & White \\
Hands-on mode without constraints & Green \\
Hands-on mode with constraints & Cyan \\
Teleoperation & Blue \\
Autonomous mode & Purple \\
Error & Red \\
\hline
\end{tabular}

However, since the color and brightness of each LED can be modified separately, the LED ring can not only visualize quasi-static robot states but also support the user dynamically in various applications, e.g. in guiding the robot's tool to a desired position using pHRI. In [18] for example, RGB-LED rings are used to insert a probe for radiofrequency ablation in a desired orientation. An implementation of this functionality could for example look like this: When starting the guidance procedure only the LED $(s)$ in the desired cartesian motion direction start(s) blinking as displayed in the right part of Fig. 4. The blinking frequency is increased when the user moves the robot towards and decreased when the user moves the robot away from the target position. Since the desired motion direction and the blinking frequency change when the robot is moving, the LED pattern is continuously updated. The arrival at the target position — within a predefined tolerance — is visualized by activating all LEDs of the ring.

\subsection{Implementation of a workflow for waterjet wound debridement}

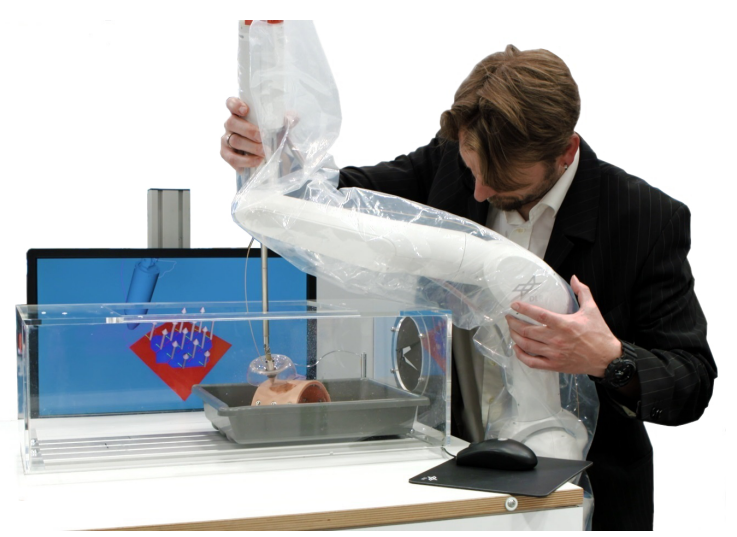

Fig. 6. Marking the area of interest for waterjet wound debridement on a silicon phantom using the robot integrated buttons of the MIRO. 
To demonstrate the potential of the presented user interface in scenarios with a single robot arm MIRO, we implemented the following workflow for robotic waterjet wound debridement ([8] compare Fig. 1 for the button denomination):

- The area of interest is marked by touching several points on the tissue surface in pHRI mode with the tip of the waterjet tool (see Fig. 6). The pHRI mode is activated by holding button B4 for several seconds and displayed by the LED ring and the robot model in the VR turning green. The selection of a point is confirmed by pressing button B4 on the robot.

- After marking a sufficient number of points around the area of interest, the capturing is finished by pressing B1 and the model of the tissue surface is created autonomously.

- Based on the surface model the path planning is performed and visualized to the user in the VR on a LCD display.

- The user moves the $M I R O$ in pHRI mode to a valid starting position and starts the autonomous execution of the wound debridement by pressing button B2. The waterjet and suction pump ERBEJET 2 ( $)$ is automatically switched on at the procedure start. If necessary, it can be switched on and off manually via button B1.

\subsection{Implementation of a workflow for the setup of a MIRS system}

As an exemplary application of the user interface in a robotic system with multiple arms, we implemented a workflow for the time-saving and intuitive system setup of our MIRS research platform DLR MiroSurge. The MiroSurge system consists of three robot arms MIRO mounted at the operating table (displayed in Fig. 1) and a surgeon console with a 3D-display and two haptic input devices sigma.7 [Force Dimension, Nyon, Switzerland] [6, 22]. One of the MIROs carries a stereo endoscope, the other two the laparoscopic robotic instruments DLR MICA. When setting up the system, at first the trocar for the endoscope is set and the endoscope is inserted. Afterwards the trocars for the instruments are set and the instruments are inserted under optical supervision from the endoscope. The implemented setup workflow comprises for each robot arm the following five steps (compare Fig. 1 for the button denomination and Fig. 7 for the setup steps):

- The user presses B1 to move the robot to a predefined approach position. The activation of the position control mode is signalized by the LED ring and the robot model in the VR turning purple (see Fig. 7(1)).

- By holding B4 the user activates the pHRI mode (displayed by green LED ring and robot model in the VR) to move the gravity compensated robot with its instrument tip to the desired trocar position as shown in Fig. 7(2).
The position of the instrument tip with regard to the robot base can be calculated from the forward kinematics of MIRO and MICA.

- Releasing B4 locks the robot's pose, allowing the user to check the chosen trocar position before confirming it via B2 (compare Fig. 7(3)). By pressing B2 the current position of the instrument tip is set as the trocar point in the workflow software of the MiroSurge system (compare Fig. 3).

- To set the trocar and to insert the instrument in the trocar, the user moves the robot in impedance control mode (activated by holding B5, LED ring and robot model in the VR turn cyan). In this mode virtual springs force the instrument axis to intersect the patient's body surface at the trocar point. Therefore the user can guide the robot with one hand while holding the trocar in the other hand as displayed in Fig. 7(4).

- When B5 is released while the instrument tip is inside the patient, the robotic system automatically switches to teleoperation mode, represented by a blue LED ring and robot model in the VR as shown in Fig. 7(5). In teleoperation mode the surgeon can teleoperate the robot from the console as demonstrated in Fig. 7(6) for the completely set-up system. If meanwhile the staff at the OR table needs better access to the patient, they can activate the nullspace motion of the robot's elbow by pressing B4.

Using the described procedure a single person (instead of the two person required before) can set up all three robots of the MIRS demonstrator MiroSurge without external user interface in less than two minutes. The described button functions and LED color assignments are exemplary and can be modified depending on the application.

Table 2. The results of the usability test for the 12 test persons. The results for the first setup procedure performed by the respective test person are written in italics. The SUS values represent the subjective user experience obtained from a standardized questionnaire (introduced in [21]) on a scale from 0 (worst possible) to 100 (best possible).

\begin{tabular}{lcccr}
\hline \multirow{2}{*}{ Test person } & \multicolumn{2}{c}{ Integrated interface } & \multicolumn{2}{c}{ No integrated interface } \\
& Setup time[s] & SUS & Setup time [s] & SUS \\
\hline 1 & 127 & 82.5 & 130 & 82.5 \\
2 & 212 & 95 & 187 & 65 \\
3 & 152 & 87.5 & 154 & 85 \\
4 & 188 & 82.5 & 130 & 65 \\
5 & 118 & 70 & 124 & 62.5 \\
6 & 232 & 90 & 157 & 72.5 \\
7 & 160 & 97.5 & 165 & 85 \\
8 & 164 & 90 & 164 & 70 \\
9 & 140 & 87.5 & 142 & 90 \\
10 & 166 & 67.5 & 135 & 75 \\
11 & 118 & 90 & 130 & 60 \\
12 & 132 & 75 & 160 & 75 \\
\hline
\end{tabular}




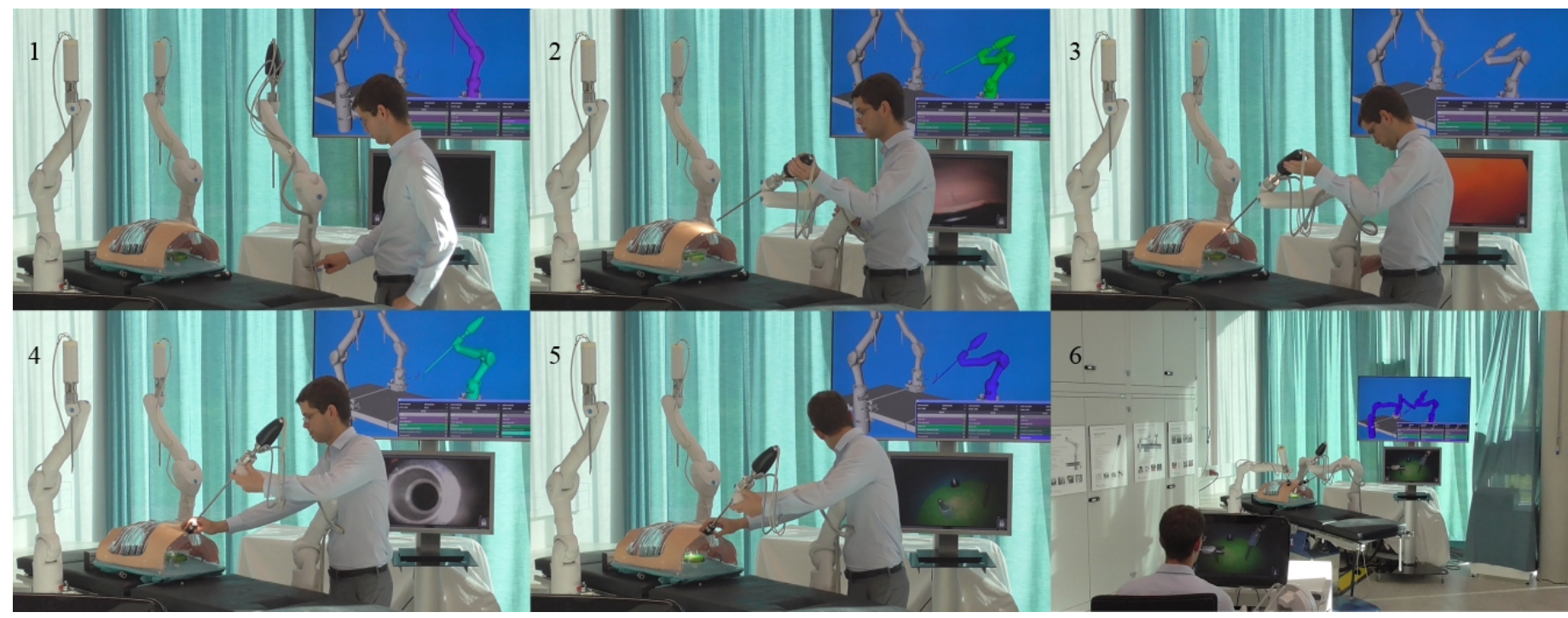

Fig. 7. The MiroSurge system with three MIRO robot arms attached to the OR table, the VR visualization of the system (large display in the upper right corner) and the endoscope image (smaller display on the right). The five steps of the implemented setup procedure are demonstrated using the endoscope robot on the right. The color of its model in the VR changes according to the control mode used in the respective setup step.: (1) move the robot in position control mode to approach position; (2) guide the instrument tip in hands-on mode to the desired trocar position; (3) confirm the selected trocar position; (4) set the trocar and insert the instrument in impedance control mode; (5) automatic activation of teleoperation mode after instrument insertion. (6) shows the teleoperation of the MiroSurge system from the user console.

\subsection{Usability test: MiroSurge setup}

Within the usability test the above described setup procedure for MiroSurge was compared to the previously used setup procedure without robot integrated buttons, where the switching of the control modes was performed by a second person - the operator — in a GUI. The measured setup times did not differ significantly with and without using the robot integrated buttons (159.1 $\mathrm{s} \pm 36.4 \mathrm{~s}$ and $148.2 \mathrm{~s} \pm 19.3 \mathrm{~s}$ respectively, $\mathrm{p}=0.2390>0.05)$. The SUS values, however, were significantly higher $(\mathrm{p}=0.0138<$ $0.05)$ for the setup procedure using the robot integrated interface $(84.6 \pm 9.5)$ than for the setup procedure not using it $(74.0 \pm 9.9)$. The setup time and SUS value for each test person are displayed in Table 2.

\section{Discussion}

The presented user interface meets the requirements formulated in section 1: It is compact and lightweight since it is completely integrated in the structure of the MIRO arm. The automatically generated functional stubs allow an easy (re)programming of buttons and LED ring in highlevel languages like $\mathrm{C}++$ or in Matlab Simulink. As shown in subsection 3.1, the interface is RT capable and allows the control of 3rd party devices.

Such an interface provides the following advantages for the robot developer:
- Low cost: The GPIO and the LED board use standard PCB technologies and electronic components. All other parts are commercially available or RP parts.

- Simple subsequent integration: The required cabling is minimized by using the existing communication infrastructure of the MIRO. The integration of the presented interfaces in another robot arm than the MIRO would require minor modifications in the mechanics (design, manufacturing and integration of RP-parts for the attachment of the buttons to the robot's structure and modification of the casings at the button and LED ring positions), electronics (connection of each GPIO board via three LVDS lines and the LED board via two LVDS lines to the communication backbone of the robot) and software (adaption of GPIO board firmware, integration of the inputs from the buttons and the output to the LEDs in the robotic middleware).

- Robust: The membranes on the casing openings enable the disinfection of the robot. For easy maintenance, the casings can be disassembled without disconnecting the button or LED cables. The mechanical robustness of the design is underlined by the fact, that it has been used in four robot arms without subsequent modifications or repairs since June 2016 (buttons) and November 2016 (LED rings) respectively. The performed research and demonstration activities also included the presentation of a scenario with pHRI for four days on the trade fair Automatica 2016 in Munich. 
- Flexible: The behavior of each RGB-LED as well as the functions of the programmable buttons can be changed without hardware modifications. As the GPIO board supports the simultaneous toggling of multiple buttons (shift function) the number of functions can surpass the number of buttons. Due to the integration in the RT communication of the robotic system the buttons can trigger besides actions of the robot itself also actions of 3rd party devices in the same RT network.

The main advantages for the robot user are:

- Usability: The buttons simplify the pHRI. The user can change the operation mode, select a workflow step or confirm an action directly at the robot. The integrated LED ring informs him/her directly about the state of the robot arm. As the robot control mode can be changed by just pushing one button, it is easily possible to adapt the number of available degrees of freedom to allow e.g. one-hand control of the robot arm whenever this is beneficial for the workflow (for example for the insertion of the MIRS instrument into the trocar). The usability test revealed that even novice users can quickly perform complex procedures like the setup of the MiroSurge platform using the robot integrated user interface: The average setup time of $159.1 \mathrm{~s}$ obtained by the 12 test persons did not that strongly exceed the $90-110 \mathrm{~s}$ we observed for trained users. The high average SUS value of 84.6 \pm 9.5 , which according to [23] corresponds to the American school grade B, underlines the good usability subjectively perceived by the 12 test persons. The average SUS value for the setup procedure with the new user interface was significantly higher than the SUS value for the same procedure without this interface $(74.0 \pm 9.9)$ : Even the novice users in the usability test, which had not worked with the user interface before, found the setup procedure with this interface subjectively easier. Provided an adequate workflow, the developed user interface apparently has the potential to increase the subjectively felt usability of the $M I R O$ or a similar lightweight robot.

- Improved multi-user operation: The interaction with the robotic system is no longer exclusively bound to additional input devices. Instead every user can interact directly with the robotic system. This is particularly advantageous in parallelizable tasks like the setup or removal of robotic systems with multiple arms, which thereby can be accelerated significantly. In the usability test, for example, the mean setup time was comparable for both setup methods. In contrast to the setup with integrated user interface, however, the setup without integrated user interface requires two persons (one user at the robot and one operator at the control desk) and therefore double the working time.

- Minimal hardware footprint: Since the robot itself serves as input device, applications like e.g. waterjet surgery require just one robot arm and one display for visualizing more complex data like e.g. the path planning.

The described integrated user interface has the po- tential to improve the handling of the MIRO or other lightweight robots especially in applications, where pHRI is required. While in the presented workflows for MIRS and waterjet wound debridement this control mode is mainly used for setting up the robotic system, it might be even more beneficial in - not necessarily exclusively medical applications, which demand a permanent close cooperation between the robotic system and its human user(s). In the medical field this is typically the case in applications where the surgeon must be continuously in full control of the procedure and simultaneously fulfill strict criteria regarding precision and/or repeatability. Examples for such kind of applications are the placement of pedicle screws [10], the insertion of a neuroendoscope [12] or the placement of a ablation probe for minimally invasive tumor treatment [18].

It should be noted, however, that in scenarios with very high accuracy requirements like e.g. neurosurgery the limited accuracy and stiffness of a lightweight robot can be insufficient. The accuracy may be increased by an appropriate calibration of the robotic arm (as e.g. described in [24]) or by cartesian optical reference control (as e.g. described in [12]). Nonetheless the robotic hardware must always be selected according to the requirements of the medical task.

In the future we want to investigate the use of the LED ring for visualizing the interaction forces of tool attached to the MIRO and the environment. The force can be measured by a commercially available force-torque sensor with digital signal output, which is connected via the GPIO board to the real-time communication of the robot. After an appropriate signal processing, these forces can be reported to the user by dynamically adapting the color of the RGB-LED ring. An user study is required to determine the practicability and accuracy of this approach.

A feasible application for such a force visualization was e.g. robotic wound debridement using a waterjet. Since in the current workflow (compare 3.2) the surface model is based on the points marked by the instrument tip, the interaction force with the typically deformable tissue should be similar for all points. An optical feedback via the LED ring would support the user in applying similar forces at each point.

\section{Conclusion}

Despite its comparatively low costs, the presented user interface is a powerful tool to operate the MIRO arm in applications with physical human robot interaction. With reasonable effort the concept can also be implemented in other lightweight robot arms like e.g. the KUKA iiwa. Provided an appropriate design of the workflow, the resulting robotic system can be operated with a minimum of accessories since each robot arm serves as its own input device and status display. The high-level control interface of the buttons and the RGB-LEDs can easily be reprogrammed for different applications. Therefore new workflows - also workflows including the control of 3rd party devices - can be quickly implemented and tested with medical staff using 
a reliable and robust interface. This rapid prototyping and instant evaluation of new workflows in user studies both accelerates the development of new medical applications and ensures the practicability of the resulting devices.

\section{References}

[1] M. Hoeckelmann, I.J. Rudas, P. Fiorini, F. Kirchner and T. Haidegger, Current capabilities and development potential in surgical robotics, Int $J$ Adv Robot Syst 12(5) (2015) 61.

[2] T.A. Mattei, A. H. Rodriguez, D. Sambhara and E. Mendel, Current state-of-the-art and future perspectives of robotic technology in neurosurgery, Neurosurg Rev 37(3) (2014) 357-366.

[3] Renishaw plc (ed), Neuromate stereotactic system Technical data sheet H-4149-0032-02-A, 2013.

[4] Intuitive Surgical Inc. (ed), da Vinci Xi System User Manual, 2014.

[5] U. Hagn, M. Nickl, S. Joerg, G. Passig, T. Bahls, A. Nothhelfer, F. Hacker, L. Le-Tien, A. Albu-Schaeffer, R. Konietschke, M. Grebenstein, R. Warpup, R. Haslinger, M. Frommberger and G. Hirzinger, The DLR MIRO: a versatile lightweight robot for surgical applications, Ind Robot 35(4) (2008) 324-336.

[6] U. Hagn, R. Konietschke, A. Tobergte, M. Nickl, S. Joerg, B. Kuebler, G. Passig, M. Groeger, F. Froehlich, U. Seibold, L. Le-Tien, A. Albu-Schaeffer, A. Nothhelfer, F. Hacker, M. Grebenstein and G. Hirzinger, DLR MiroSurge - A Versatile System for Research in Endoscopic Telesurgery, Int J Comput Assist Radiol Surg 5(2) (2010) 183-192.

[7] S. Haddadin, A. Albu-Schaeffer and G. Hirzinger, Requirements for safe robots: Measurements, analysis and new insights, IJRR 28(11-12) (2009) 1507-1527.

[8] T. Bahls, F. Froehlich, A. Hellings, B. Deutschmann and A. Albu-Schaeffer, Extending the Capability of Using a Waterjet in Surgical Interventions by the Use of Robotics, IEEE Transactions on Biomedical Engineering 64(2) (2017) 284-294.

[9] F. Froehlich, G. Passig, A. Vazquez and G. Hirzinger, Robot assisted internal mammary artery detection for coronary revascularisation surgery, in Proc. IROS 2010 (Taiwan, Taipei, 2010), pp. 1849-1855.

[10] T. Ortmaier, H. Weiss, U. Hagn, M. Grebenstein, M. Nickl, A. Albu-Schaeffer, C. Ott, S. Joerg, R. Konietschke, L. Le-Tien and G. Hirzinger, A hands-onrobot for accurate placement of pedicle screws, in Proc. ICRA 2006 (USA, Orlando, 2006), pp. 41794186.

[11] R. Konietschke, T. Ortmaier, C. Ott, U. Hagn, L. LeTien and G. Hirzinger, Concepts of human-robot co- operation for a new medical robot, in Proc. of the second international workshop on human centered robotic systems (HCRS), (2006), pp. 1-6.

[12] M. Niccolini, V. Castelli, C. Diversi, B. Kang, F. Mussa and E. Sinibaldi, Development and preliminary assessment of a robotic platform for neuroendoscopy based on a lightweight robot, Int J Med Robotics Comput Assist Surg 12 (2016) 4-17.

[13] E. Guizzo and E. Ackerman, How Rethink Robotics built its new Baxter robot worker, in IEEE Spectrum (2012).

[14] E. Guizzo, Rethink Robotics' Sawyer Goes on sale, Rodney Brooks says 'There May Be More Robots', in IEEE Spectrum (2015).

[15] KUKA AG (ed), LBR iiwa - Teaching by demonstration (2014), https://www.youtube.com/watch?v=r7gU74Yv9Es.

[16] Mazor Inc. (ed), Introducing Mazor $X^{T M}: A$ Surgical Assurance Platform (2016), https://www.youtube.com/watch?v=wdecPXTxW50.

[17] C. Bergeles and G-Z. Yang, From passive tool holders to microsurgeons: safer, smaller, smarter surgical robots, IEEE Transactions on Biomedical Engineering 61(5) (2014) 1565-1576.

[18] M. Herrlich, J. Benker, D. Black, F. Dylla and R. Malaka, Tool-mounted ring displays for intraoperative navigation, in Tagungsband der 14. Jahrestagung der Deutschen Gesellschaft fuer Computerund Roboterassistierte Chirurgie (CURAC), eds. H.K. Hahn et al. (Germany, Bremen, 2015), pp. 273-278.

[19] S. Joerg, M. Nickl, A. Nothhelfer, T. Bahls and G. Hirzinger, The Computing and Communication Architecture of the DLR Hand Arm System, in Proc. IROS 2011 (San Francisco, USA, 2011), pp. 1055-1062.

[20] M. Nickl, T. Bahls, A. Nothhelfer and S. Strasser, SpaceWire, a backbone for humanoid robotic systems, in Proc. of the 4th International SpaceWire Conference (Dundee, UK, 2011), pp. 356-359.

[21] J. Brooke, SUS-A quick and dirty usability scale, Usability evaluation in industry 189(194) (1996) 4-7.

[22] A. Tobergte and A. Albu-Schaeffer, Direct force reflecting teleoperation with a flexible joint robot, in Proc. ICRA 2012 (USA, Minnesota, St.Paul, 2012), pp. $4280-4287$.

[23] A. Bangor, P.Kortum and J. Miller, Determining what individual SUS scores mean: Adding an adjective rating scale, Journal of Usability studies 4(3) (2009) 114123.

[24] J. Klodmann, R. Konietschke, A. Albu-Schaeffer and G. Hirzinger, Static Calibration of the DLR Medical Robot MIRO, a Flexible Lightweight Robot with Integrated Torque Sensors, in Proc. IROS 2011 (San Francisco, USA, 2011), pp. 3708-3715. 